\title{
Assessment of antimutagenic avenue and wild plant diversity on roadside near Nature Park, Kolkata, India
}

\author{
Subhashis Das, Kushal Banerjee, Abantika Nandy, \\ Soumendra Nath Talapatra* \\ Department of Environmental Science, University of Calcutta, \\ 35 Ballygunge Circular Road, Kolkata 700019, India \\ *Phone: 91-9831008689 \\ *E-mail address: ecologylive@yahoo.co.in
}

\begin{abstract}
The study of diversity of plants in an urban area is a suitable measure of the terrestrial ecosystem. The study of avenue and wild plants diversity is an important research work, which provides knowledge about naturally occurring chemicals in plants, to protect against mutation, cancer and other diseases. These are commonly called as phytochemicals and the natural chemical ingredients are potent to use in antimutagenicity. The present study aims to understand the occurrence and population nos. of different avenue and wild plants planted or natural on roadside near Nature Park, Kolkata, India. The results clearly indicate that a total population of these plant species are having 282 nos. in the study area. Various literatures clearly revealed that these plants and/or their parts have potent antimutagenic properties by the presence of several phytochemicals. In conclusion, present preliminary research emphasizes an occurrence of antimutagenic avenue and wild plants on roadsides, containing natural chemical ingredients. Previous research works confirmed that toxins or mutagens or carcinogens may cause mutation that leads to cancer. The present study is a preliminary assessment of antimutagenic avenue and wild plant species diversity that have not yet been studied in that particular geographical area. The present study also suggested that avenue and wild plants are suitable for antimutagenic potential along with aesthetical view of human as well as ecosystem support. Therefore, biodiversity study, phytochemicals estimation and conservation of these particular plant species and/or different antimutagenic species in other local area viz. roadsides, parks, forests etc. might be relevant because the plant species can be used for herbal medicine in human healthcare as well as to prevent mutation and cancer.
\end{abstract}

Keywords: Avenue and wild plant diversity; Plant diversity; Anti-mutagenic plants; Medicinal plants; Biodiversity

\section{INTRODUCTION}

Plants produce naturally occurring antimutagenic compounds viz. flavonoids, phenolics, coumarins, carotenoids, antraquinones, tannins, saponins, etc. that have protective effects against mutagens. Natural antimutagens from edible and medicinal plants are of particular importance because they are preventive for human cancer (Sanjib, 2011) and also 
genotoxicity prevention (Talapatra et al. 2010). Even for populations using herbs traditionally, encouraging the use of species with chemopreventive actions could be helpful as a part of life expectancy improvement strategies: costs are significantly low, herbs have usually little or no toxicity during long-term oral administration and are relatively available at large scale (Satish et al., 2013). It has been suggested that regular consumption of anticarcinogens and antimutagens in the diet may be the most effective way of preventing human cancer and search for novel antimutagens acting in chemoprevention is a promising arena in phytotherapy (Gowri and Chinnaswamy, 2011). According to Turner et al. (2007), biodiversity study has been shown to be inequitably distributed across cities, potentially due to micro level variation in vegetation and other biological resources. Ministry of Environment and Forests (MoEF) has prescribed avenue trees for the aesthetic views and air pollution control. Although roadside trees perform ecological functions such as sequestering carbon, reducing summer cooling costs, removing airborne pollutants, and controlling stormwater runoff (Rowntree and Nowak 1991; McPherson 1994; Nowak 1994; Qi et al. 1998; Beckett et al. 2000). Beside these, avenue trees and other common plant species have potent antimutagenic properties (Agarwal and Pandey, 2009; Satwinderjeet et al. 2010; Sanjib, 2011; Satish et al., 2013; Espanha et al., 2014; Joselin et al., 2014). Numerous studies from four decades have been out in order to identify compounds, which might protect humans against DNA-damage and its consequences (DeFlora and Ramel, 1988). Many plant species are known to elicit antimutagenes is and thus have a full range of prospective applications in human healthcare (Satish et al., 2013). The diversity of different plant species as avenue trees, peripheral trees for greenbelt, trees in particular park etc. have already been documented nationally and internationally (Benthal, 1946; Chakraverty and Jain, 1984; McPherson and Rowntree, 1989; Galvin, 1999; Mukhopadhyay and Chakraverty, 2008; Zainudin et al., 2012) but no one has attempted to study the diversity of antimutagenic avenue trees and other plant species on both sides of the road. The present study aims to know the qualitative and quantitative assessment of antimutagenic plant diversity of avenue and wild plant species located on roadside near Nature park, Kolkata, India.

\section{MATERIALS AND METHODS}

The study area was selected at both sides of the road near Nature park (latitude $=22^{\circ}$ $31^{\prime} \mathrm{N}$ and longitude $=88^{\circ} 17^{\prime} \mathrm{E}$ ), Kolkata, India. The field study was carried out in the month of August 2014 and September 2014 (monsoon season). The qualitative and quantitative assessment was done by 900 meter line transect and the antimutagenic plant species variety and individual number of species was evaluated as described by the methods of Jaenson et al. (1992). Field survey was done by identifying and counting the plant species and visual identification and finally image capture in this study.

The diversity of avenue and wild plant species were studied by qualitative and quantitative assessment as antimutagenic species. The usage of parts of the plants and their antimutagenic phytochemicals were studied from various literatures and tabulated. The number of each species observed was tabulated and statistical analysis was carried out using Microsoft Excel sheets. Relative abundances were calculated and tabulated separately for individual plant species. Species richness, Index of Dominance, Shannon-Wiener Diversity Index, and Evenness Index for both plant species for above-mentioned selected sites were calculated by using the following statistical formulae (Stiling, 1999): 
Relative abundance (Pi)

$\mathrm{Pi}=\mathrm{Ni} / \mathrm{N}$

Where, $\mathrm{Ni}$ is the number of Individuals of a species, and $\mathrm{N}$ is total population of plants.

Species Richness $(\mathrm{d})=\mathrm{S}-1 / \ln \mathrm{N}$

Where, $\mathrm{S}=$ number of species, $\ln \mathrm{N}=$ natural logarithm of the total number of individuals.

Index of Dominance $(\mathrm{C})=\sum(\mathrm{ni} / \mathrm{N})^{2}$

Where, $\mathrm{ni}=$ importance value for each species (number of individuals), $\mathrm{N}=$ total number of importance value

Shannon-Wiener diversity index $\left(H^{\prime}\right), \mathrm{H}^{\prime}=-\left[\sum \mathrm{Pi} \ln \mathrm{Pi}\right]$

Where, $\mathrm{Pi}$ is proportion of species $\mathrm{i}$ relative to the total number of species, and $\ln \mathrm{Pi}$ is natural logarithm of this proportion.

Evenness index Species Evenness $=\mathrm{H}^{\prime} / \ln (\mathrm{S})$

Where, H' is Shannon Diversity Index; $\mathrm{S}$ is Species Richness (number of species), and ln (S) is natural logarithm of species Richness.

\section{RESULTS}

The present results clearly indicate that diversity of roadside plants as avenue and wild plants near Nature park and their parts contain potent antimutagenic properties as phytochemicals after studying from various literatures (Table 1).

Table 1. Qualititaive and quantitative assessment of anti-mutagenic avenue and wild plant diversity near Nature park, Kolkata.

\begin{tabular}{|c|c|c|c|c|c|c|c|c|}
\hline $\begin{array}{c}\text { Sl. } \\
\text { No. }\end{array}$ & $\begin{array}{l}\text { Type } \\
\text { of } \\
\text { plants }\end{array}$ & $\begin{array}{c}\text { Plant } \\
\text { species } \\
\text { (common } \\
\text { name) }\end{array}$ & $\begin{array}{c}\text { Plant } \\
\text { species } \\
\text { (scientific } \\
\text { name) }\end{array}$ & $\begin{array}{c}\text { No. of } \\
\text { individual } \\
\text { species }\end{array}$ & $\begin{array}{c}\text { Relative } \\
\text { abundan } \\
\text { ce }(\%)\end{array}$ & $\begin{array}{c}\text { Part (s) } \\
\text { used }\end{array}$ & $\begin{array}{l}\text { Antimutagenic } \\
\text { compounds }\end{array}$ & $\begin{array}{c}\text { Literatures } \\
\text { Referred }\end{array}$ \\
\hline 1. & $\underset{\mathscr{D}}{\mathscr{E}}$ & 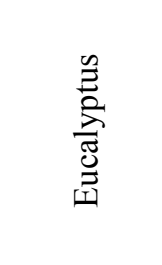 & 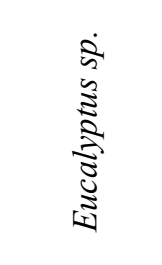 & $\curvearrowright$ & $\stackrel{\hat{0}}{\circ}$ & 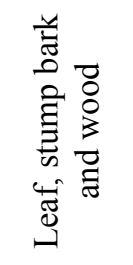 & 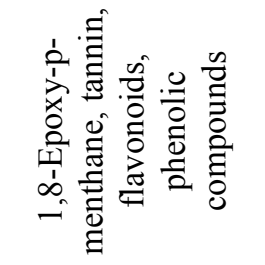 & 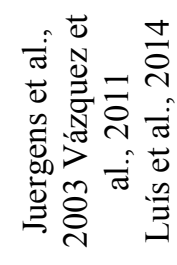 \\
\hline 2. & 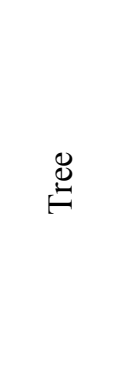 & 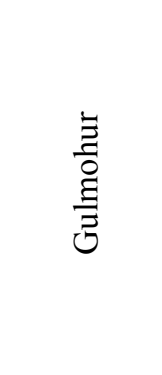 & 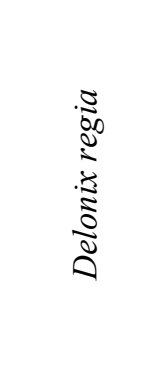 & $\widehat{\sim}$ & : & 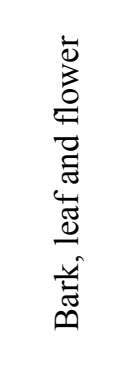 & 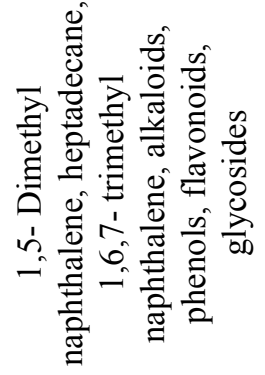 & 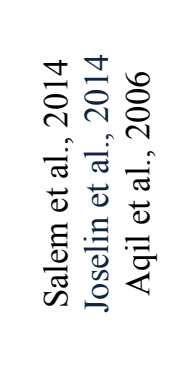 \\
\hline
\end{tabular}




\begin{tabular}{|c|c|c|c|c|c|c|c|c|}
\hline 3. & 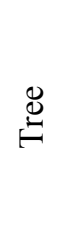 & 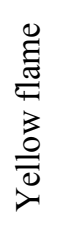 & 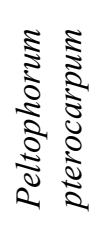 & $\stackrel{f}{f}$ & $\begin{array}{l}0 \\
\stackrel{0}{0}\end{array}$ & 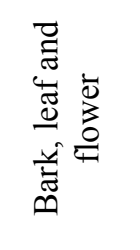 & 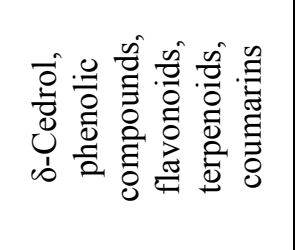 & 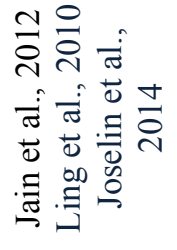 \\
\hline 4. & 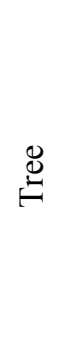 & 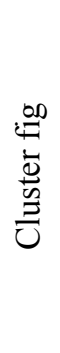 & 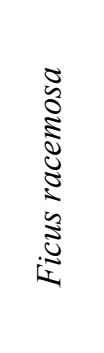 & $\hat{n}$ & $\begin{array}{l}\infty \\
\infty \\
\stackrel{0}{0}\end{array}$ & 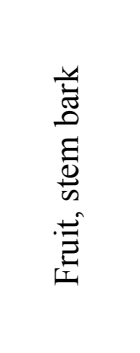 & 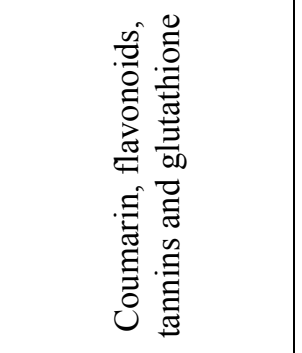 & 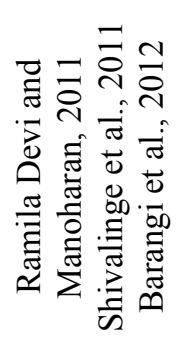 \\
\hline 5. & $\underset{\mathscr{E}}{\mathscr{E}}$ & 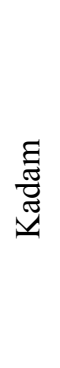 & 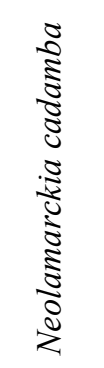 & $\stackrel{0}{\sim}$ & $\begin{array}{l}\text { ò } \\
\text { o }\end{array}$ & 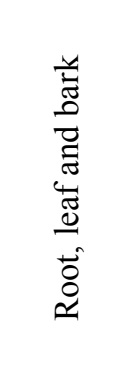 & 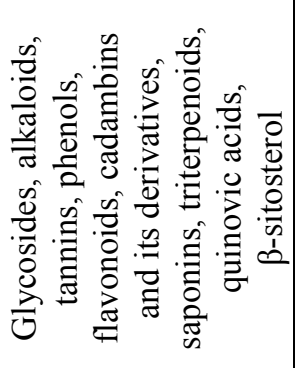 & 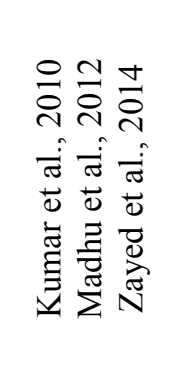 \\
\hline 6. & 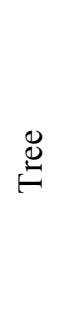 & $\begin{array}{l}\text { D } \\
\text { E } \\
\sum\end{array}$ & 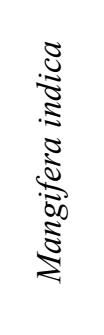 & $\tilde{\sigma}$ & $\stackrel{0}{0}$ & 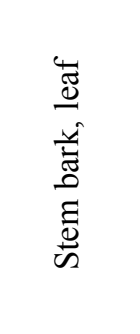 & 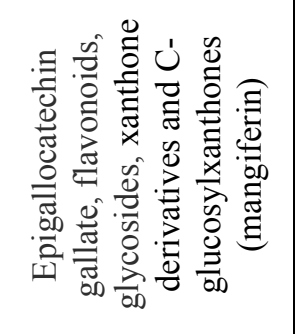 & 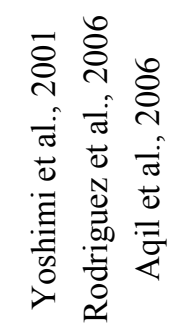 \\
\hline 7. & 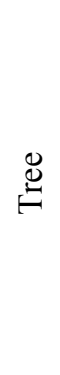 & 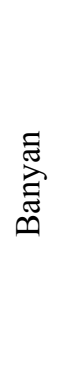 & 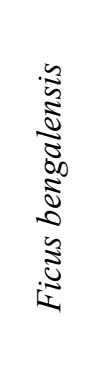 & $\ddot{\theta}$ & $\stackrel{\infty}{\circ}$ & 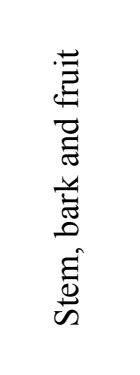 & 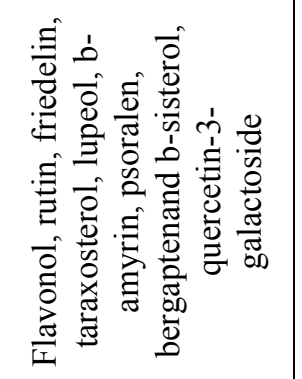 & 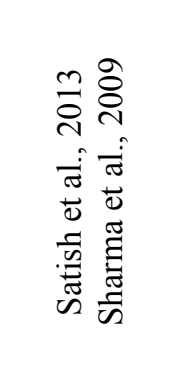 \\
\hline 8. & 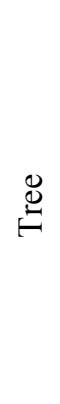 & 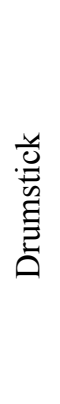 & 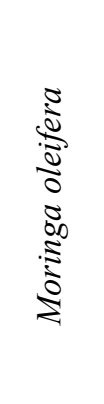 & $\delta$ & $\begin{array}{l}1 \\
\stackrel{8}{0}\end{array}$ & 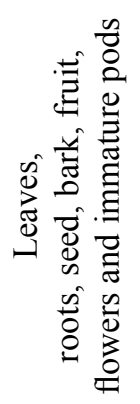 & 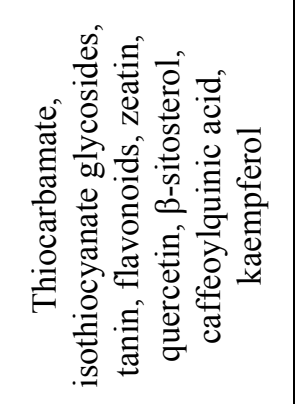 & 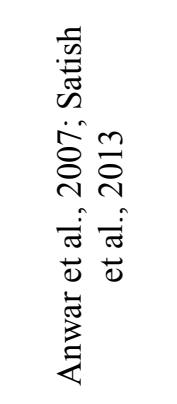 \\
\hline
\end{tabular}




\begin{tabular}{|c|c|c|c|c|c|c|c|c|}
\hline 9. & 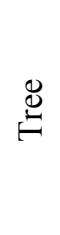 & 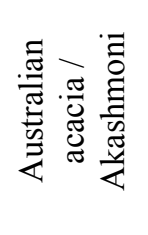 & 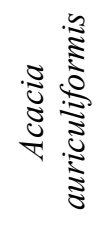 & $\hat{o}$ & 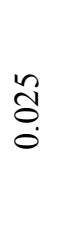 & 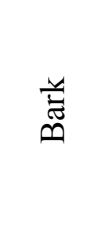 & 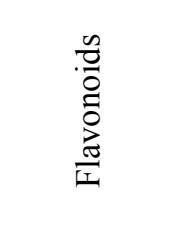 & 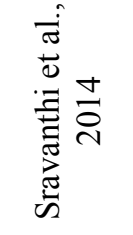 \\
\hline 10. & $\underset{\Xi}{\mathscr{E}}$ & $\begin{array}{l}\overline{0} \\
\text { ō } \\
\text { o. }\end{array}$ & 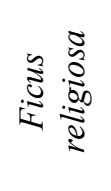 & 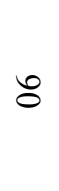 & $\begin{array}{l}\overrightarrow{\widehat{O}} \\
\stackrel{0}{0}\end{array}$ & 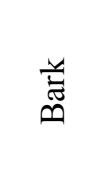 & 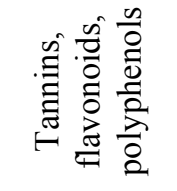 & 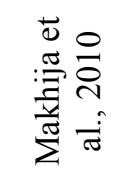 \\
\hline 11. & $\underset{\mathscr{E}}{\stackrel{\Xi}{\rightleftarrows}}$ & 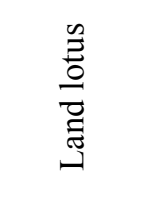 & 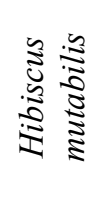 & $\bar{\sigma}$ & $\begin{array}{l}\text { ôे } \\
\dot{0}\end{array}$ & 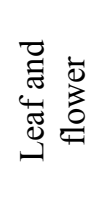 & $\begin{array}{l}\frac{n}{0} \\
0 \\
0 \\
0 \\
\frac{\pi}{L}\end{array}$ & 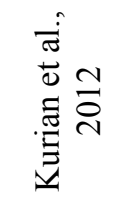 \\
\hline 12. & 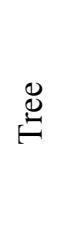 & 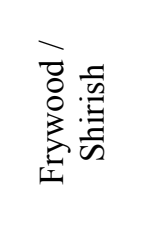 & 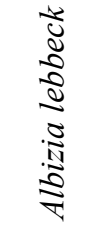 & 8 & है & $\begin{array}{l}\vec{D} \\
\mathscr{D} \\
\mathscr{n}\end{array}$ & 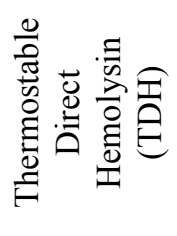 & 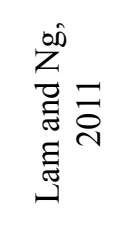 \\
\hline 13. & $\underset{\mathscr{E}}{\mathscr{\Xi}}$ & 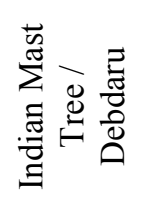 & 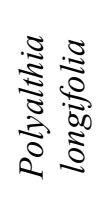 & ธิ & $\begin{array}{l}1 \\
\stackrel{8}{0} \\
\stackrel{0}{0}\end{array}$ & $\stackrel{\widetilde{J}}{\Theta}$ & 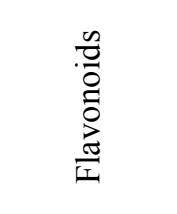 & 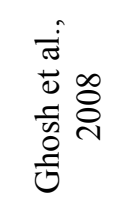 \\
\hline 14. & 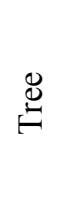 & 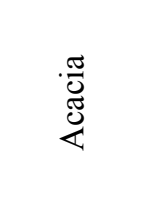 & 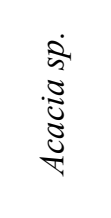 & 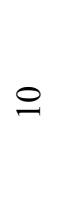 & $\begin{array}{l}\text { חे } \\
0 \\
0\end{array}$ & z & 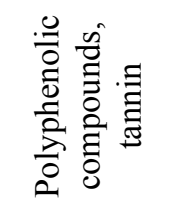 & 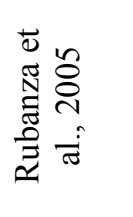 \\
\hline 15. & $\underset{\mathscr{\Xi}}{\mathscr{\Xi}}$ & 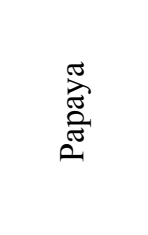 & $\begin{array}{l}0 \\
0 \\
0 \\
0 \\
0 \\
0 \\
0 \\
0\end{array}$ & $\tilde{0}$ & $\vec{\Xi}$ & 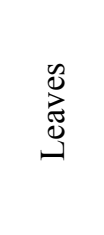 & 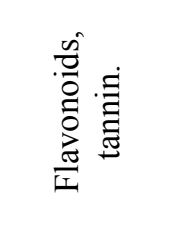 & 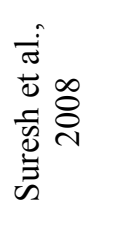 \\
\hline 16. & 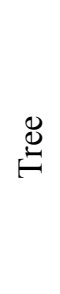 & 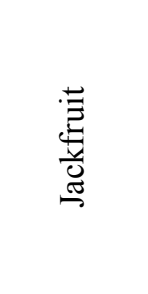 & 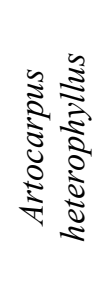 & ธิ & $\stackrel{1}{0}$ & 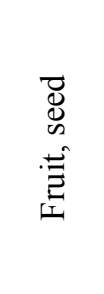 & 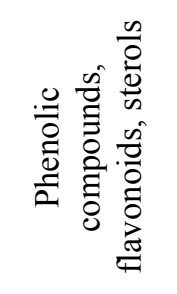 & 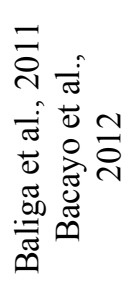 \\
\hline 17. & 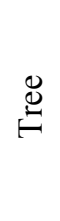 & 帚 & 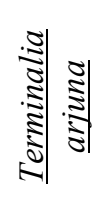 & $\widehat{\imath}$ & $\begin{array}{l}\text { \&े } \\
0 \\
0\end{array}$ & 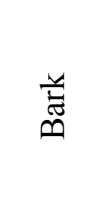 & $\begin{array}{l}\frac{n}{0} \\
0 \\
0 \\
0 \\
\frac{\pi}{I}\end{array}$ & 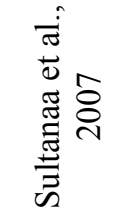 \\
\hline
\end{tabular}




\begin{tabular}{|c|c|c|c|c|c|c|c|c|}
\hline 18. & $\stackrel{\mathscr{\Xi}}{\stackrel{\mathscr{Z}}{*}}$ & $\begin{array}{l}\cdot \frac{\pi}{u} \\
0 \\
0 \\
0 \\
3 \\
0 \\
0 \\
0\end{array}$ & 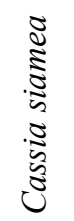 & $\delta$ & $\vec{z}$ & $\begin{array}{l}\frac{n}{0} \\
\frac{\pi}{2} \\
\stackrel{0}{0} \\
\overline{0} \\
\frac{0}{1} \\
\frac{0}{I}\end{array}$ & 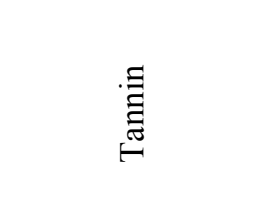 & 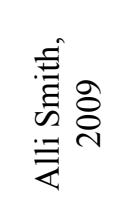 \\
\hline 19. & $\underset{\mathscr{E}}{\mathscr{\Xi}}$ & 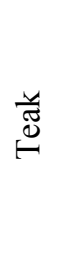 & 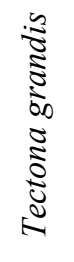 & 0 & $\begin{array}{l}\tilde{n} \\
0 \\
0\end{array}$ & 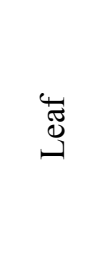 & 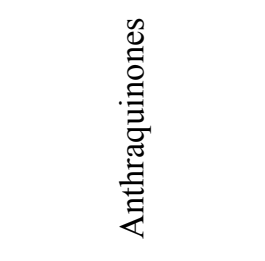 & 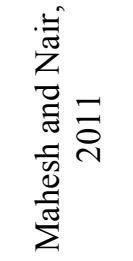 \\
\hline 20. & 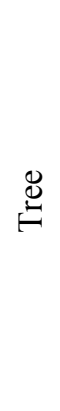 & 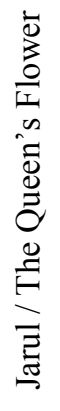 & 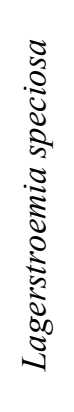 & t & $\begin{array}{l}\stackrel{\Delta}{0} \\
\stackrel{0}{0}\end{array}$ & 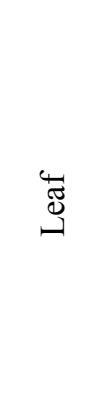 & 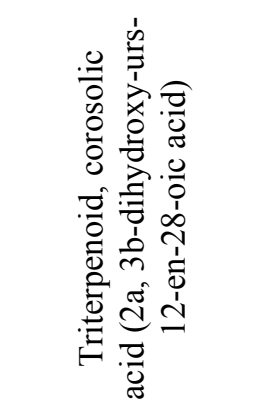 & 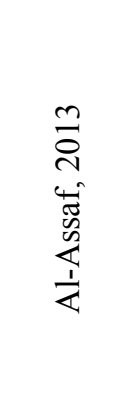 \\
\hline 21. & $\underset{\mathscr{\Xi}}{\mathscr{\Xi}}$ & $\dot{\bar{\nu}}$ & 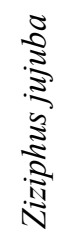 & $\bar{\sigma}$ & $\begin{array}{l}\tilde{o} \\
0 \\
0\end{array}$ & 奇 & 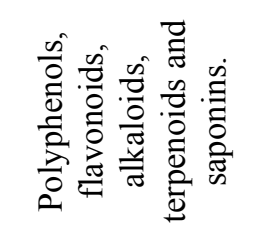 & 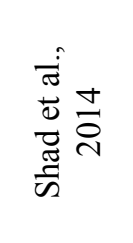 \\
\hline 22 & $\stackrel{\mathscr{ٌ}}{\mathscr{E}}$ & 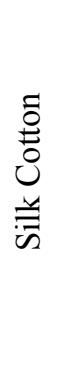 & 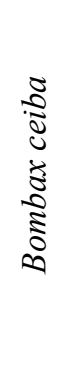 & ô & $\begin{array}{l}\stackrel{2}{0} \\
\stackrel{0}{0}\end{array}$ & 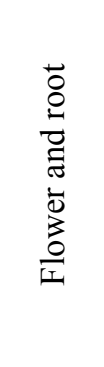 & 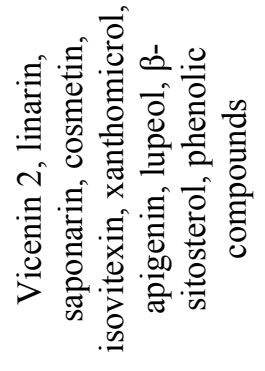 & 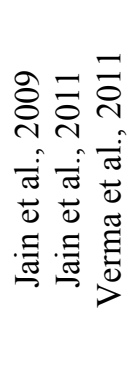 \\
\hline 23. & 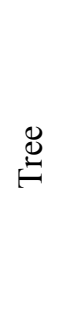 & 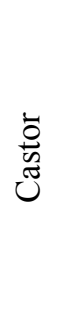 & 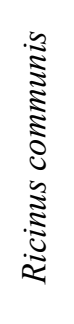 & t & 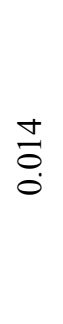 & 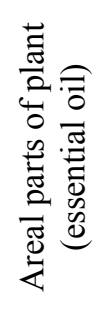 & 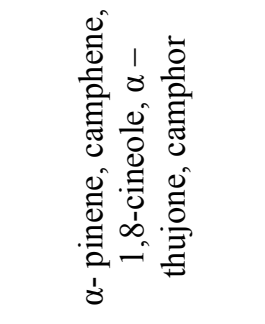 & 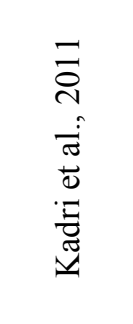 \\
\hline
\end{tabular}

In the roadside plants, total number of species was observed 282 in numbers, in which 19 nos. of Eucalyptus sp., 27 nos. of Delonix regia, 46 nos. of Peltophorum pterocarpum, 53 nos. of Ficus racemosa, 26 nos. of Neolamarckia cadamba, 02 nos. of Mangifera indica, 05 nos. of Ficus bengalensis, 02 nos. of Moringa oleifera, 07 nos. of Acacia auriculiformis, 06 
nos. of Ficus religiosa, 01 no. of Hibiscus mutabilis, 09 nos. of Albizia lebbeck, 02 nos. of Polyalthia longifolia, 10 nos. of Acacia sp., 3 nos. of Carica papaya, 2 nos. of Artocarpus heterophyllus, 27 nos. of Terminalia arjuna, 03 nos. of Cassia siamea, 16 nos. of Tectona grandis, 04 nos. of Lagerstroemia speciosa, 01 no. of Ziziphus jujuba, 07 nos. of Bombax ceiba, 04 nos. of Ricinus communis (Table 1).

It was studied and recorded from various literatures that the different parts of plants were also containing phytochemicals to protect mutagenecity (Table 1). In Eucalyptus sp., leaf, stump bark and wood contains 1,8-epoxy-p-menthane, tannin, flavonoids and phenolic compounds, in Delonix regia, bark, leaf and flower contains 1,5-dimethylnaphthalene, heptadecane, 1,6,7- trimethyl napthalene, alkaloids, phenols, flavonoids and glycosides, in Peltophorum pterocarpum, bark, leaf and flower contains $\delta$-cedrol, phenolic compounds, flavonoids, terpenoids and coumarins, in Ficus racemosa, fruits, stem and bark contain coumarin, flavonoids, tannins and glutathione, in Neolamarckia cadamba, root, leaf and bark contains glycosides, alkaloids, tannins, phenols, flavonoids, cadambins and its derivatives, saponins, triterpenoids, quinovic acids, and $\beta$-sitosterol, in Mangifera indica stem, bark and leaves contain epigallocatechin gallate, flavonoids, glycosides, xanthone derivatives and $\mathrm{C}$ glucosylxanthones (mangiferin), in Ficus bengalensis, stem, bark and fruit contain flavonol, rutin, friedelin, taraxosterol, lupeol, $\beta$-amyrin, psoralen, bergaptenand $\beta$-sisterol and quercetin-3-galactoside, in Moringa oleifera, leaves, roots, seed, bark, fruit, flowers and immature pods contain thiocarbamate, isothiocyanate glycosides, tanin, flavonoids, zeatin, quercetin, $\beta$-sitosterol, caffeoylquinic acid and kaempferol, in Acacia auriculiformis, bark contains flavonoids, in Ficus religiosa, fruit contains tannins, flavonoids and polyphenols, in Hibiscus mutabilis, leaf and flower contains flavonoids, in Albizia lebbeck, seed contains thermostable direct hemolysin (TDH), in Polyalthia longifolia, leaf contains flavonoids, in Acacia sp., pod contains polyphenolic compounds and tannin, in Carica papaya leaves contain flavonoids and tannin, in Artocarpus heterophyllus, fruits and seeds contain phenolic compounds, flavonoids and sterols, in Terminalia arjuna, bark contains flavonoids, in Cassia siamea, flower petals contain tannin, in Tectona grandis, leaf contains anthraquinones, in Lagerstroemia speciosa leaf contains triterpenoid and corosolic acid (2a, 3b-dihydroxy-urs12-en-28-oic acid), in Ziziphus jujube, fruits contain polyphenols, flavonoids, alkaloids, terpenoids and saponins., in Bombax ceiba, flower and root contain vicenin 2, linarin, saponarin, cosmetin, isovitexin, xanthomicrol, apigenin, lupeol, $\beta$-sitosterol and phenolic compounds and in Ricinus communis, areal parts of plant (essential oil) contain $\alpha$ - pinene, camphene, 1,8-cineole, $\alpha$-thujone and camphor.

In Table 1, the relative abundance value of each species was tabulated such as Eucalyptus sp. 0.067, Delonix regia 0.096, Peltophorum pterocarpum 0.163, Ficus racemosa 0.188, Neolamarckia cadamba 0.092, Mangifera indica 0.007, Ficus bengalensis 0.018, Moringa oleifera 0.007, Acacia auriculiformis 0.025, Ficus religiosa 0.021, Hibiscus mutabilis 0.003, Albizia lebbeck 0.032, Polyalthia longifolia 0.007, Acacia sp. 0.035, Carica papaya 0.011, Artocarpus heterophyllus 0.007, Terminalia arjuna 0.096, Cassia siamea 0.011, Tectona grandis 0.057, Lagerstroemia speciosa 0.014, Ziziphus jujube 0.003, Bombax ceiba 0.025 , Ricinus communis 0.014 respectively.

Different diversity indices for antimuatagenic avenue and wild plants were calculated (Table 2), species richness was found to be 0.078 . For index of dominance, a value of 0.102 was observed. For Shanon-Weiner diversity index, 2.582 was calculated. In case of evenness index, it was found 0.823 (Table 2). 
Table 2. Study of different indices for antimutagenic avenue and wild plant diversity.

\begin{tabular}{|c|c|c|}
\hline Sl. No. & Different indices & Values \\
\hline 1. & Species richness & 0.078 \\
\hline 2. & Index of dominance & 0.102 \\
\hline 3. & Shanon-Weiner Diversity index & 2.582 \\
\hline 4. & Evenness index & 0.823 \\
\hline
\end{tabular}

\section{DISCUSSION}

The present study of antimutagenic plants diversity near Nature park indicates that these avenue and wild plants contain potent phytochemicals. This study is a preliminary observation on roadside avenue as well as wild plants having antimutagenic potential and can be used in herbal medicine.

Biodiversity study has been shown to be inequitably distributed across cities, potentially due to micro level variation in vegetation and other biological resources (Turner et al., 2007). The plants diversity study in parks, forests, industrial vicinity, roadsides etc. (Schroeder and Cannon, 1983; Ulrich, 1985; Heisler, 1986; Dwyer et al., 1992; Cumming et al., 1997; Canon et al., 1998; Nowak et al., 2000; Nowak and Crane, 2002; Xiao and McPherson, 2002; Ragasa et al., 2009; Chawla et al., 2012; Zainudin et al., 2012) and plant species individually as potent antimutagenic, anticarcinogenic, antioxidant, antitumour containing chemical ingredients have already been studied nationally and internationally (Agarwal and Pandey, 2009; Satwinderjeet et al. 2010; Sanjib, 2011; Satish et al., 2013; Talapatra, 2013; Espanha et al., 2014; Joselin et al., 2014). But very scanty research work has been carried out on antimutagenic potential by avenue plants and their parts (Joselin et al., 2014) and no one has attempted to study the diversity of avenue plants in Kolkata, India, which can be used as antimutagenic herbal drugs.

Although biodiversity of several medicinal plants have already been documented by many researchers (Samant and Dhar, 1997; Khan et al., 2009; Lal and Singh, 2012; Talapatra, 2013). The whole plants and/or parts of the plants of herbs, shrubs and trees contain several secondary metabolites, which have been utilized in medicinal practice. The villagers use them as folk medicine by their traditional knowledge.

In Table 1, the plant diversity near Nature park, Kolkata, India was studied and tabulated. The present study showed the varieties of avenue and wild plants that have potent antimutagenic phytochemicals after detailed study of various literatures. It was observed that highest numbers of plant species were 53 nos. of Ficus racemosa and lowest number was 01 no. of Ziziphus jujuba and Hibiscus mutabilis, followed by in a descending order such as 46 nos. of Peltophorum pterocarpum, 27 nos. of Delonix regia and Terminalia arjuna, 26 nos. of Neolamarckia cadamba, 19 nos. of Eucalyptus sp., 16 nos. of Tectona grandis, 10 nos. of Acacia sp., 09 nos. of Albizia lebbeck, 07 nos. of Acacia auriculiformis and Bombax ceiba, 06 nos. of Ficus religiosa, 05 nos. of Ficus bengalensis, 04 nos. of Lagerstroemia speciosa and Ricinus communis, 3 nos. of Carica papaya and Cassia siamea, 02 nos. of Moringa oleifera, Mangifera indica, Artocarpus heterophyllus and Polyalthia longifolia. The highest 
percentage of relative abundance value was observed 0.188 in Ficus racemosa while lowest value was 0.003 in Ziziphus jujuba and Hibiscus mutabilis.

Various literatures clearly revealed that medicinal plants have well been studied on the various chemical contents as phytochemicals in the whole plants and/or parts of the plants. In Table 1, the parts of the avenue and wild plants and their potent active phytochemicals as antimutagenic and/or anti-carcinogenic and/or antioxidant properties were tabulated from various research works. It was studied that Eucalyptus sp. has potent phytochemicals like 1,8-Epoxyp-menthane, tannin, flavonoids and phenolic compounds found in leaves, stump barks and woods (Juergens et al., 2003; Vázquez et al., 2011; Luís et al., 2014). In Delonix regia, bark, leaf and flower contains 1,5- dimethyl naphthalene, heptadecane, 1,6,7- trimethyl napthalene, alkaloids, phenols, flavonoids and glycosides. These phytochemicals have been identified and estimated by Aqil et al., 2006; Salem et al., 2014 and Joselin et al., 2014. According to Ling et al., 2010; Jain et al., 2012; Joselin et al., 2014, the Peltophorum pterocarpum, bark, leaf and flower contains $\delta$-cedrol, phenolic compounds, flavonoids, terpenoids and coumarins. In all ficus species were recorded such as Ficus racemosa, the fruits, stem and bark contain coumarin, flavonoids, tannins and glutathione (Ramila Devi and Manoharan, 2011; Shivalinge et al., 2011; Barangi et al., 2012), Ficus bengalensis, the stem, bark and fruit contain flavonol, rutin, friedelin, taraxosterol, lupeol, $\beta$-amyrin, psoralen, bergaptenand $\beta$ sisterol and quercetin-3-galactoside (Sharma et al., 2009; Satish et al., 2013) and Ficus religiosa, the fruit contains tannins, flavonoids and polyphenols (Makhija et al., 2010). It was studied in Neolamarckia cadamba, the root, leaf and bark contains glycosides, alkaloids, tannins, phenols, flavonoids, cadambins and its derivatives, saponins, triterpenoids, quinovic acids, and $\beta$-sitosterol (Kumar et al., 2010; Madhu et al., 2012; Zayed et al., 2014).

According to Yoshimi et al., 2001; Rodriguez et al., 2006; Aqil et al., 2006, the common plant, Mangifera indica, the stem, bark and leaves contain epigallocatechin gallate, flavonoids, glycosides, xanthone derivatives and C-glucosylxanthones (mangiferin). In Moringa oleifera, the leaves, roots, seed, bark, fruit, flowers and immature pods contain thiocarbamate, isothiocyanate glycosides, tanin, flavonoids, zeatin, quercetin, $\beta$-sitosterol, caffeoylquinic acid and kaempferol (Anwar et al., 2007; Satish et al., 2013). A common avenue plants, Acacia auriculiformis and Terminalia arjuna, the bark of both species contains flavonoids (Sravanthi et al., 2014 and Sultanaa et al., 2007) and Polyalthia longifolia, the leaves contain flavonoids (Ghosh et al., 2008) while other plant species Hibiscus mutabilis, leaves and flowers both contain flavonoids (Kurian et al., 2012). According to Lam and Ng, (2011), the avenue plant, Albizia lebbeck, the seed contains thermostable direct hemolysin (TDH). In Acacia sp., pod contains polyphenolic compounds and tannin (Rubanza et al., 2005), the Carica papaya leaves contain flavonoids and tannin (Suresh et al., 2008), in Artocarpus heterophyllus, fruits and seeds contain phenolic compounds, flavonoids and sterols (Baliga et al., 2011; Bacayo et al., 2012). Other avenue plant species, Cassia siamea, flower petals contain tannin (Alli Smith, 2009), Tectona grandis, leaf contains anthraquinones (Mahesh and Nair, 2011), Lagerstroemia speciosa leaf contains triterpenoid and corosolic acid or 2a, 3b-dihydroxy-urs-12-en-28-oic acid (Al-Assaf, 2013), Bombax ceiba, flower and root contain vicenin 2, linarin, saponarin, cosmetin, isovitexin, xanthomicrol, apigenin, lupeol, $\beta$-sitosterol and phenolic compounds (Jain et al., 2009; Jain et al., 2011; Verma et al., 2011). The wild species such as Ziziphus jujube, fruits contain polyphenols, flavonoids, alkaloids, terpenoids and saponins (Shad et al., 2014) and Ricinus communis, areal parts of plant (essential oil) contain $\alpha$ - pinene, camphene, 1,8-cineole, $\alpha$-thujone and camphor (Kadri et al., 2011). 
For Shanon-Weiner diversity index, a value of 2.582 was observed, which indicate high diversity of antimutagenic plant species (Table 2). To know the diversity of antimutagenic plant species as an opportunity to obtain phytochemicals from avenue and wild plants' origin, further research work might be helpful in relation to medicinal plant diversity in other roadsides, parks, local forests along with the protection of plant species and their phytochemicals for the usage in herbal medicine. As there is no previous study of this avenue and wild plant species on the antimutagenic potential with specific tree species at this particular geographical area.

\section{CONCLUSIONS}

In the present study it was concluded that the variety and numbers of avenue and wild plant species are suitable for antimutagenic potential by their natural chemical ingredients as phytochemicals (Joselin et al., 2014). As we know from the previous research work that toxins or mutagens or carcinogens may cause mutation that leads to cancer (Yoshimi et al., 2001; Talapatra et al., 2014). This study is a preliminary assessment of antimutagenic avenue and wild plant species diversity that have not yet been studied in the particular geographical area. The present study also suggested that avenue plants are suitable for antimutagenic potential along with aesthetical view of human as well as ecosystem support. Therefore, biodiversity study, phytochemicals estimation and conservation of these particular species and/or other antimutagenic species in other local area viz. roadsides, parks, forests etc. might be relevant and the plant species can be used for herbal medicine for human healthcare and also to prevent mutation and cancer.

\section{Acknowledgement}

The authors convey their gratitude to Dr. Snehasikta Swarnakar, Principal Scientist, CSIR-Indian Institute of Chemical Biology, Drug Development Diagnostic \& Biotechnology Division, for providing critical comments in manuscript. The authors also thankful to the local people during field study by their continuous help and identification of the plant species by local name.

\section{References}

[1] Agarwal R.C., Pandey S., Asian Pacific Journal Cancer Prevention 10 (2009) 913-916.

[2] Al-Assaf A.H., Pakistan Journal of Nutrition 12 (8) (2013) 748-752.

[3] Alli Smith Y.R., Pakistan Journal of Nutrition 8 (2) (2009) 119-121.

[4] Anwar, F., Latif, S., Ashraf, M. And Gilani, A.H. Moringa oleifera: a food plant with multiple medicinal uses. Phytotherapeutic Research 21 (2007) 17-25.

[5] Aqil F., Ahmed I., Mehmood Z., Turkish Journal of Biology 3 (2006) 177-183.

[6] Bacayo M.F.D., Sydney Paole Anne G., Ceniza S.P.A.G., Montaño S.M.M., Ubalde R.A.V., Root Gatherers 3 (2012) 123-130. 
[7] Baliga M.S., Shivashankara A.R., Haniadka R., Dsouza J., Harshith P., Bhat H.P., Food Research International 44 (8) (2011) 1800-1811.

[8] Barangi S., Vidya S.M., Sanjeev G., Rao V.C., Rajesh K.P., Vinutha K.J., Biochemical Technology 3(5) (2012) S212-S217.

[9] Beckett K.P., Freer-Smith P., Taylor G., J Arboric 26 (2000) 12-19.

[10] Benthal A.P., The Trees of Calcutta and its Neighbourhood. Thacker Spink and Co. Ltd., Calcutta (1946).

[11] Cannon C.H., Peart D.R., Leighton M., Science 28 (1998) 1366-1368.

[12] Chakraverty R. K. and Jain S. K. (1984). Beautiful Trees and Shrubs of Calcutta. Botanical Survery of India.

[13] Chawla A., Yadav P.K., Uniyal S.K., Kumar A., Vats S. K., Kumar S. and Ahuja P.S., Current Science 102 (8) (2012) 1143-1156.

[14] Cumming D.H.M., Fenton M.B., Rautenbach I.L., Taylor R.D., Cumming G.S., Cumming M.S., Dunlop J.M., Ford A.G., Hovorka M.D., Johnston D.S., Kalcounis M., Mahlangu Z., Portfors C.V.R., South African J Sci 93 (1997) 231-236.

[15] DeFlora S., Ramel C., Mutat Res 202 (1988) 285-306.

[16] Espanha L.G., Resende F.A. Neto J.S.L., Boldrin P.K., Nogueira C.H., de Camargo M.S., De Grandis R.A., dos Santos L.C., Vilegas W., Varanda E.A., BMC Complementary and Alternative Medicine 14 (2014) 182.

[17] Dwyer J. F., McPherson E. G., Schroeder H.W. and Rowntree R. A., J Aboric 18(5) (1992) 227-234.

[18] Galvin M.F., J. Arboric 25 (1999) 124-128.

[19] Ghosh A., Das B.K., Chatterjee S. K., Chandra G., The South Pacific Journal of Natural Science 26 (2008) 68-72.

[20] Gowri S., Chinnaswamy P., J Nat Prod Plant Resour 1 (2011) 27-34.

[21] Heisler G.M., J Aboric 12(5) (1986) 113-125.

[22] Jaeson R., Bassuk N., Schawer S., Headley D., J Arboric 18(4) (1992) 171-183.

[23] Jain S.C., Pancholi B., Jain R., Der Pharma Chemica 4 (5) (2012) 2073-2079.

[24] Jain V., Verma S.K., Katewa S.K., Indian journal of traditional Knowledge 8(4) (2009) 638-644.

[25] Jain V., Verma S.K., Katewa S.K., Anandjiwala S., Singh B., Research Journal of Medicinal Plant 5(4) (2011) 462-470.

[26] Joselin J., Florence A.R., Brintha T.S.S., Jeeva S., Journal of Chemical and Pharmaceutical Research 6(7) (2014) 2089-2096.

[27] Juergens U.R., Dethlefsen U., Steinkamp G., Gillissen A., Repges R., Vetter H., Respiratory Medicine 97 (2003) 250 -256.

[28] Kadri A., Gharsallah N., Damak M., Gdoura R., Journal of Medicinal Plants Research 5(8) (2011) 1466-1470. 
[29] Khan M., Kumar S., Hamal I.A., Ethnobotanical Leaflets 13 (2009) 1113- 1139.

[30] Kumar V., et al., Indian J Biochemistry Biophysics 47 (2010) 104-109.

[31] Kurian J.C., J Biodiversity 3(1) (2012) 61-75.

[32] Lal H. S., Singh S., Bioscience Discovery 3(1) (2012) 91-96.

[33] Lam S. K., Ng T. B., Phytomedicine 18(7) (2011) 601-608.

[34] Ling L.T., Radhakrishnan A.K., Subramanian T., Cheng H.M., Palanisamy D.P. Molecules 15 (2010) 2139-2151.

[35] Luís A., Neiva D., Pereira H., Gominho J., Domingues F., Duarte A.P., Molecules 19 (2014) 16428-16446.

[36] Mahesh S.K., Nair J.A., International Journal of Phytomedicine 3 (2011) 50-58.

[37] Makhija I.K., Sharma I.P., Khamar D., Annals of Biological Research 1(4) (2010) 171-180.

[38] Madhu C., Sharma U., Kumar N., Singh B., Satwinderjeet K., Asian Pacific Journal of Tropical Medicine 5(12) (2012) 977-985.

[39] McPherson E.G., Energy-saving potential of trees in Chicago, pp 95-113. In McPherson E.G., DJ. Nowak, and R.A.Rowntree (Eds.). Chicago's Urban Forest Ecosystem: Results of the Chicago Urban Forest Climate Project, General Technical Report NE-186. USDA Forest Service, Northeastern Forest Experiment Station, Radnor, PA (1994).

[40] McPherson E.G., Rowntree R.A., Landscape Journal 8 (1989) 13-23.

[41] Mukhopadhyay D.P., Chakraverty R.K. Plant wealth of the Raj Bhavan Kolkata, Occasional Paper - 5 from Raj Bhavan, Kolkata, pp.1-163 (2008)..

[42] Nowak D.J., Atmospheric carbon dioxide reduction by Chicago's urban forest, pp 83-94. In McPherson E.G., DJ. Nowak, and R.A. Rowntree (Eds). Chicago's Urban Forest Ecosystem: Results of the Chicago Urban Forest Climate Project, General Technical Report NE-186. USDA Forest Service, Northeastern Forest Experiment Station, Radnor, PA (1994).

[43] Nowak D. J., Civerolo K.L., Rao S.T., Sistla G., Luley C.J., Crane D.E., Atmospheric Environment 34 (2000) 1601-1613.

[44] Nowak D.J., Crane D.E., Environmental Pollution 116 (2002) 381-389.

[45] Qi Y., Favorite J., Lorenzo A., Forestry: A Community Tradition. National Association of State Foresters. Joint Publication of USDA Forest Service, the National Association of State Foresters and Southern University and A and M College. $3^{\text {rd }}$ Edition, pp. 35 (1998).

[46] Ragasa C.Y., Tsai Po-W, Shen C-C., Philippine Journal of Science 138 (2) (2009) 205-209.

[47] Ramila Devi M., Manoharan A., J Chem Pharm Res 3(6) (2011) 166-172. 
[48] Rodriguez J., Pierro D. D., Gioia M., Monaco S., Delgado R., Coletta M., Marini S., Biochimica et Biophysica Acta 1760(9) (2006) 1333-1342.

[49] Rowntree R.A., Nowak D.J., J Arboric 17 (1991) 269-275.

[50] Rubanza C., Shem M., Otsyina R., Bakengesa S., Ichinohe T., Fujihara T., Anim Feed Sci Technol 119 (2005) 129-142.

[51] Salem M.Z.M., Ahmed A.M., Ali H.M., Bioresources 9(2) (2014) 2382-2395.

[52] Samant S.S., Dhar U., Int J Sustain Dev World Ecol 4 (1997) 179-191.

[53] Sanjib B., Res J Med Plant 5 (2011) 116-126.

[54] Satish A., Kumar R.P., Rakshith D., Satish S., and Ahmed F., International Journal of Chemical and Analytical Science 4 (2013) 45-48.

[55] Satwinderjeet K., Subodh K., Prabhjit K., Madhu C., Am J Biomed Sci 2 (2010) 164-177.

[56] Schroeder H.W., Cannon W.N., J Aboric 9(9) (1983) 237-243.

[57] Sharma R.K., Chatterji S., Rai D.K., Mehta S., Rai P.K., Singh R.K., Watal G., Sharma B., Journal of Medicinal Plants Research 3(11) (2009) 944-948.

[58] Shad A.A., Ahmad S., Ullah R., AbdEl-Salam N.M., Fouad H., NajeebUr Rehman N. U., Hussain H., Saeed W., Scientific World Journal (2014) 1-7. http://dx.doi.org/10.1155/2014/857363

[59] Shivalinge K.P., Vrushabendra B.M., Int J Res Pharma Chem 1(3) (2011) 465-469.

[60] Sravanthi S., Ch.Santosh Madan Mohan M., Journal of Environmental and Applied Bioresearch 2(1 ) (2014) 1-4.

[61] Stiling P., Ecology: Theories and Applications (3 ${ }^{\text {rd }}$ ed) (1999) Upper Saddle River, NJ: Prentice-Hall, Inc.

[62] Sultanaa B., Anwara F., Przybylski R., Food Chemistry 104 (3) (2007) 1106-1114.

[63] Suresh K., Deepa P, Harisaranraj R., Vaira A.V., Ethnobotanical Leaflets 12 (2008) 1184-1191.

[64] Talapatra S.N., Dasgupta S., Guha A., Auddy M., Mukhopadhyay A., Food Chem Toxicol 48(12) (2010) 3458-3461.

[65] Talapatra S.N., Local medicinal plants of Maheshtala, Kolkata, India. www.youtube.com/watch?v=mv5kjgMfrMI. Nov 29 (2013).

[66] Talapatra S.N., Banerjee P., Mukhopadhyay A., International Letters of Natural Sciences 4 (2014) 36-43.

[67] Turner W.R., Landscape Urban Plann 65 (2002) 149-166.

[68] Turner W.R., Brandon K., Rooks T.M., Robert Costanza R., da Fonseca G.A.B., Portela R., BioScience 57(10) (2007) 868-873.

[69] Ulrich R.S. Landscape and Urban Planning 13 (1985) 29-44.

[70] Vázquez G., Santos J., Freire M., Antorrena G., González-Álvarez J., Wood Sci Technol 46 (2011) 443-457. 
[71] Verma V., Jalalpure S.S., Sahu A., Bhardwaj L.K., Prakesh Y., Internationale Pharmaceutica Sciencia 1 (1) (2011) 62-68.

[72] Xiao Q., McPherson E.G., Urban Ecosystems 6 (2002) 291-302.

[72] Yoshimi N., Matsunaga K., Katayama M., Yamada Y., Kuno T., Qiao Z., Hara A., Yamahara J., Mori H., Cancer Letters 163 (2001) 163-170.

[73] Zainudin S.R., Mustafa K.A., Austin D., Helmy J., Lingkeu D.A., J Trop Agric Sci 35 (1) (2012) 27-32.

[74] Zayed M.Z., Ahmad F.B., Ho W-S., Pang S-L., International Journal of Pharmacy and Pharmaceutical Sciences 6 (9) (2014) 123-127. 